\title{
Qué enseñamos y cómo enseñamos a investigar. El caso del posgrado en Humanidades, énfasis en estudios sobre artes de la Universidad de Sonora
}

\author{
Leonel De Gunther Delgado \\ leonel.degunther@unison.mx \\ https://orcid.org/0000-0002-8075-0818 \\ Diana Brenscheidt genannt Jost \\ diana.brenscheidt@unison.mx \\ https://orcid.org/0000-0003-3916-1963 \\ Universidad de Sonora \\ Gloria Clemencia Valencia González \\ gvalencia@ucm.edu.co \\ Universidad Católica de Manizalez, Colombia.
}

\begin{abstract}
¿Por qué los humanos hacen ciencia? ¿por qué arte? Las cosas menos importantes para la supervivencia es lo que nos hace verdaderamente humanos. Savas Dimopoulos
\end{abstract}

Introducción

El objetivo de este trabajo consiste en mostrar los supuestos que subyacen a la enseñanza de la investigación en el posgrado en Humanidades, estudios en artes de la Universidad de Sonora. Plantea como pregunta central qué enseñamos y cómo enseñamos a investigar en el entramado de los distintos modos de saber disponibles sea en las ciencias naturales, las ciencias sociales, las humanidades y las artes, así como también, en las ciencias de la complejidad sea restringida o no restringida y en los estudios culturales (Morin, 2008; Wallerstein, 2002); tal pregunta, en esta ocasión, la colocamos en la dimensión del profesorado como uno de los actores de este proceso. Abordamos la investigación como una esfera de la actividad humana (Bajtin, 1999) generada al interior de la universidad o los centros de investigación públicos o privados, cuyo aprendizaje, en el ámbito educativo, demanda constancia, dedicación y esfuerzo para conciliar un proceso constructivo sujeto del error y la ilusión humana (Morin, 1999). Para su desarrollo seguimos como orientador metódico, los supuestos de la reflexibilidad metodológica expuestos por Piovani (2016), los cuales nos permiten actualizar una hipótesis de trabajo enunciada por Bruschi: una metodología sin reflexión sobre los fines, condiciones y límites del conocimiento es sólo una tecnología o práctica fuera del control de quien la ejerce, mientras que, si abandona el lado tecnológico o práctico, se convierte en reflexión filosófica, sin injerencia en las actividades de investigación. De ahí que, a toda actividad metódica (técnico-práctica) subyazcan soluciones metodológicas y, a estas, respuestas a cuestiones epistemológicas (como se cita en Marradi, Archenti, y Piovani, 2007) y, agregamos a estas, ontológicas, las cuales tienen consecuencias en nuestras formas de conocer. Se trata de comprender las razones o supuestos que empleamos para dirigir los procesos de enseñanza y 
de aprendizaje de la metodología de la investigación. Tal proceso reflexivo lo construimos quienes participamos tanto en la enseñanza de la investigación como en la dirección de tesis de posgrado de este programa. Entre los principales hallazgos destacamos que es necesario alcanzar un equilibrio entre la dimensión instrumentalizadora de la enseñanza de la investigación y el de la recreación de la misma a través del conocimiento de los supuestos que subyacen a ella. Una tarea, sin duda, compleja.

La investigación en la

universidad

La investigación como una esfera de la actividad humana (Bajtin, 1999), generada por la universidad, los centros de investigación públicos o privados y diferenciada de otras actividades al interior de la universidad, constituye una tarea que concluye con la escritura y publicación de un artículo en una revista científica (Mari Mut, 2013) o en una "editorial de prestigio" o más aún, cuando se publica en un Journal indexado bajo un proceso de revisión por pares (López, Alvarado, y Mungaray, 2011; Vargas, 2009) o, si se prefiere, cuando el trabajo haya sido citado por algún colega en algún trabajo del mismo tipo.

La afirmación anterior conlleva un sinnúmero de tareas que bien pueden iniciar con la búsqueda de convocatorias, la planificación inicial del proyecto, la búsqueda de recursos, la revisión bibliográfica, el trabajo de fichado, la organización crítica de la información, la exploración y diálogo con los textos revisados, la contextualización y construcción del problema de investigación, la elección o construcción adecuada del método, el diseño de la investigación y de los instrumentos para la recogida o construcción de los "datos", el análisis y discusión de los resultados, las conclusiones y el cuidado de la bibliografía empleada son sólo algunas de las actividades que consumen el tiempo del investigador, sin señalar los tiempos de espera y las correcciones que siempre ocurren entre la aceptación del texto y su publicación.

Como un punto intermedio entre lo dicho hasta ahora, hablamos además de un dominio de conocimiento específico sobre la confección de la investigación y agregamos también, su comunicación adecuada a través de la escritura académica que, como sabemos, descansa en el conocimiento de la lengua, en el dominio de la gramática en sentido amplio, en el conocimiento de géneros y de estilos asociados a las disciplinas y sus contextos de producción. Es decir, en el reconocimiento explícito de la investigación como una actividad social y culturalmente situada y su escritura como un instrumento para aprender los contenidos y formas de pensamiento disciplinares y un medio para promover el diálogo entre comunidades internacionales como señala Castelló (2014).

La breve enunciación expresada más arriba describe, en parte, el trabajo que cotidianamente realizamos para el desarrollo de la investigación en la universidad; sin embargo, tal descripción subsume los supuestos $\mathrm{u}$ orientaciones que subyacen a cada uno de esos aspectos y, también, cómo son hechos explícitos al momento de su enseñanza y aprendizaje. De ahí que, nuestro propósito en este trabajo consista en mostrar los supuestos u orientadores que subyacen a la enseñanza de la investigación en el posgrado en Humanidades, estudios en artes de la Universidad de Sonora.

Planteamos como pregunta central qué enseñamos y cómo enseñamos a investigar en el entramado de los distintos modos de saber disponibles sea en las ciencias naturales, las ciencias sociales, las humanidades y las artes, así como también, en las ciencias de la complejidad sea restringida o no restringida y en los es- 
tudios culturales (Morin, 2008; Wallerstein, 2002), tal pregunta la colocamos en la dimensión del profesorado como uno de los actores de este proceso. Para ello, elaboramos un ejercicio de reflexividad metodológica entendido como

... lo que significa el proceso de investigación desde un punto de vista metodológico... que incluye el examen de los supuestos de tales métodos, de los procesos socio-históricos implicados en su construcción y aceptación, y de su relación con diferentes posicionamientos teóricos y epistemológicos (Piovani, 2016, p. 4747).

En tal ejercicio no descuidamos, siguiendo a Leal (2003), la noción de crítica concebida tanto como discernir, discriminar entre un sistema de pensamientos mejor fundado que otro y también, dicho con cautela, como el establecimiento de los límites y alcances de nuestras decisiones y posicionamientos sobre la enseñanza de la metodología de la investigación.

A continuación, damos cuenta de los supuestos que subyacen a la enseñanza de la investigación.

\section{Los supuestos de la enseñanza de la metodología de la investigación.}

Nuestro punto de partida para la enseñanza de la investigación comienza con la revisión del Proyecto de posgrado en Humanidades (Universidad de Sonora, 2008), en él se expresan de manera general las funciones y finalidades educativas que orientan tanto los estudios de doctorado como de maestría y sus énfasis específicos ${ }^{1}$, aquí solo nos referiremos al énfasis en artes.

La pregunta principal que dirige el desa-

\footnotetext{
1 Sea Maestría en Lingüística, Maestría en Literatura Hispanoamericana y los programas: Especialización en Enseñanza de Lenguas Extranjeras, Especialización en Energía y Medioambiente en Arquitectura, Maestría en Humanidades, Doctorado en Humanidades y Doctorado Humanidades como programa integral.
}

rrollo curricular del programa de posgrado alude a la herencia del conocimiento disponible, la cual colocada en las artes plantea ${ }^{2}$ ¿ ¿cuál es el estado de desarrollo que guardan las disciplinas artísticas en la formación de los distintos profesionistas en México y en el mundo en general? (Universidad de Sonora, 2008, p. 10), una pregunta enorme, cuyas respuestas posibles --sin desarrollar en el documento-- ponen en el centro de la reflexión al ser humano y su bienestar en sus múltiples dimensiones sea económica, de salud física, mental, emocional o cognitiva (Universidad de Sonora, 2008, p. 10), cuya comprensión sirva para transformar nuestras concepciones sobre lo que sea el hombre y la ciencia y haga emerger que el problema principal que hoy afronta la humanidad es el de su supervivencia como especie. Se trata entonces de alcanzar "una mayor comprensión de los seres humanos y de las culturas, mismas que, aunque ajenas, no dejan de ser apartados de la humanidad" (Universidad de Sonora, 2008, p. 10).

De ahí que el programa se adhiera, sin descuidar sus marcos disciplinares, a una visión inter- o trans- disciplinaria e intente responder otras preguntas particulares ${ }^{3}$ : ¿Cuál debe ser o debería ser el impacto de las artes en el desarrollo del conocimiento actual? ¿Cuál es el estatus del arte, su vigencia e impacto? ¿Cuál debe ser o debería ser el peso de las artes en el modelo de desarrollo social, educativo y cultural?

\footnotetext{
2 La pregunta original es: "cuál es el estado de desarrollo que guardan las disciplinas humanísticas en la formación de los distintos profesionistas en México y en el mundo en general". (p. 10).

3 ¿Cuál debe ser o debería ser el impacto de las Humanidades en el desarrollo del conocimiento actual? ¿Cuál debe ser o debería ser el peso de las Humanidades en el modelo de desarrollo social, educativo y cultural? ¿Cuál debería ser la visión Humanista en el estado de desarrollo de las tecnologías? ¿Cuál debería ser la aportación de las Humanidades en términos de la explotación de los recursos naturales? (p. 10) ¿Cuál debería ser el papel de las Humanidades en la conservación y mejoramiento del medio ambiente? (p. 11).
} 
¿Cuál debería ser la visión de las artes en el estado de desarrollo de las tecnologías? ¿Cuál debería ser la aportación de las artes en términos de la explotación de los recursos naturales? ¿Cuál debería ser el papel de las artes en la conservación y mejoramiento del medio ambiente? (Universidad de Sonora, 2008, pp. 10-11), preguntas que, si bien resultan pertinentes, requieren de investigaciones específicas para su resolución, además de que colocan la investigación en las artes tanto en el ámbito de la explicación como de la comprensión. No sólo se trata, como señalan Pérez y Rodríguez (2011), de cuestiones relativas a la causalidad, sino a la comprensión de las razones que subyacen a nuestras acciones, todo desde una orientación empírica.

Las preguntas expresadas más arriba constituyen, siguiendo a Gil Anton (2004), intereses cognitivos, útiles para la elaboración de conceptos, sea en la explicación o comprensión de fenómenos, es decir, de fenómenos no interpretables o interpretables: En el primero de los casos se trata de preguntas cuyo interés se centra en el establecimiento de regularidades donde el esquema causa efecto conduce a la explicación; mientras que en las segundas, la interpretación dota de sentido o permiten comprender la regularidad. En cualquiera de los casos, se trata de preguntas que bien pueden quedarse tanto en la disciplina o articular disciplinas. Una pregunta como ¿Cuál debe ser o debería ser el peso de las artes en el modelo de desarrollo educativo? Es de esa clase.

Tales preguntas se convierten entonces en los grandes tópicos o lugares comunes de la investigación humanística del posgrado, donde el lenguaje adquiere un énfasis especial como mediación en la producción del conocimiento. Bosquejan, además, las posibles interacciones con otras ciencias y disciplinas desde un enfoque humanístico orientado a la reflexión y/o solución de problemas sociales y culturales en el entorno regional y nacional. De este breve marco enunciativo se desprende que

"la investigación en las áreas de humanidades afronta la función de promover en la sociedad el análisis, la comprensión y la valoración de las opciones de cambio y cursos de acción posibles y deseables, promoviendo la reflexión sistemática sobre los grandes problemas nacionales y locales y aportando estudios críticos sobre la sociedad actual" (Universidad de Sonora, 2008, p. 12).

Finalmente, la estructura curricular del programa incluye tres áreas básicas: la de formación disciplinar, la de formación humanística y la de formación metodológica o de investigación, esta última de nuestro interés.

El resumen curricular del apartado anterior constituye el primer supuesto que guía la enseñanza de la investigación y lo denominamos "orientación curricular". Esta orientación nos permite colocar los procesos de investigación tanto en los ámbitos disciplinares como expandir sus posibilidades hacia los estudios inter- 0 trans- disciplinares. Tales supuestos pueden fundamentarse en los planteamientos de Wallerstein (2007); Wallerstein et al., (1996), Morin, (1983, 2008) y García (2006).

Se trata, por un lado, de modos de saber, incorporados en campos de conocimiento con disciplinas afines, cuyos procesos de especialización e hiperespecialización si bien necesarios, obstaculizan la comprensión de los fenómenos sociales, culturales y humanos en una época cada vez más compleja. Se trata de formas de conocer, distribuidas en los diferentes campos de conocimientos, que establecen marcos para la investigación; pero, a la vez, impiden la adquisición de nuevos conocimientos, los llamados obstáculos epistemológicos señalados por Bachelard 
(2000); y por el otro, la irrupción de los estudios culturales y de la complejidad como respuesta crítica a las lógicas positivistas y pos-positivistas incorporadas en la disciplinas, dirigiendo su mirada hacia otras dimensiones de lo real que habían permanecido ocultas o al margen de los intereses "científicos", nos referimos aquí a la teoría crítica o los planteamientos constructivistas y, en el caso de la complejidad a la comprensión del tejido conjunto de los fenómenos y, a la búsqueda de una comprensión lo menos reductora posible de la realidad y, dicho sea esquemáticamente, al planteamiento de García (2006), en el cual se elige poner al frente los problemas humanos y, bajo ellos, las disciplinas como soporte de su resolución.

En cualquiera de los casos, se trata de formas de saber que tienen que ver, dicho sociológicamente, con las formas de producción, organización y distribución del conocimiento disperso y disponible sea en los ámbitos de las ciencias naturales, sociales y de las humanidades y las artes, así como también a las tradiciones de investigación, en los procesos formativos de especialistas e hiper-especialistas e, incluso, en la formación de generalistas y, también, de sus finalidades. Esos planteamientos generan pautas para la investigación, que abrevan desde las dimensiones ontológicas, epistemológicas y metodológicas, las cuales tienen implicaciones en los productos de la investigación.

De lo anterior se desprende el segundo supuesto: el reconocimiento y conocimiento del campo de conocimiento y de las disciplinas afines donde se ubica el investigador/a y de un posicionamiento crítico sobre lo que sea la realidad, las maneras que tenemos para acceder a ella, los procedimientos disponibles para hacerlo y, también, a los principios de inclusión o exclusión entre ellos. Tal supuesto implica un entrenamiento no instrumental en la comprensión de las pautas que sub- yacen a nuestras formas de conocer, que abren un espacio a la argumentación y a la creatividad del investigador/a para la toma de decisiones específicas en su investigación.

Un ejemplo de lo anterior lo podemos extraer tanto de los textos de Guba and Lincoln (1994) como en los de Cortés (2008), los cuales aluden a las consecuencias de las orientaciones que guían al investigador en su proyecto, cuyos fundamentos ontológico: lo constituyente del mundo; epistemológico: la posibilidad de conocer el mundo, y metodológicos: procedimientos sistemáticos; los cuales se van perfilando como si fueran una función de los supuestos que gobiernan su práctica de investigación y de su construcción metódica, lo sepa o no el investigador (su paradigma).

Así, para decirlo de manera esquemática y con precaución ${ }^{4}$, una elección sobre la forma y la naturaleza de la realidad y sobre lo que podamos conocer de ella, determina las preguntas que podemos formular. Por ejemplo, si suponemos la existencia del mundo real (como lo hace el pos-positivismo), las preguntas formulables son aquellas relacionadas a la existencia de acciones reales; de tal suerte que el universo de la ética o estética quedan fuera de la investigación legítima. De la misma manera, la naturaleza de la relación entre el que conoce y lo que busca conocer queda determinada por la ontología supuesta por el investigador. Siguiendo la lógica del ejemplo planteado, el investigador buscará el distanciamiento objetivo y libre de valores, lo cual le permitirá decir lo que las cosas son y cómo realmente funcionan. Ante las preguntas metodológicas, sobre cómo puede conocer lo que

4 Valles (2009), ofrece un posicionamiento crítico a la versión de Guba y Lincoln derivado de lo esquemático y simplista de la misma. De la misma manera, han surgido también nuevos planteamientos con respecto a las formas de conocer, actualmente, Gabriel (2015), con su planteamiento sobre el nuevo realismo viene a cuestionar también estos posicionamientos. 
supone el investigador puede ser conocido, el acotamiento metodológico está dado sobre los supuestos ontológicos y epistemológicos. Si lo que está en juego es la objetividad, habría entonces que exigir el control de variables extrañas sea en métodos cuantitativos o cualitativos y orientar la investigación hacia el empleo de métodos que permitan la manipulación o experimentación, la pluralidad crítica, la triangulación, etc.; en este sentido, "la pregunta metodológica no puede reducirse a una pregunta acerca de los métodos; los métodos deben adecuarse a una metodología predeterminada" (Guba y Lin coln, 1994, p. 108).

El ejemplo anterior es válido si nos colocamos en la teoría crítica o en el constructivismo, lo que cambia son las consecuencias derivadas a partir de los supuestos ontológicos, epistemológicos y metodológicos en el proceder de nuestra investigación. La cuestión de la investigación también cambia si nos adentramos en otras lógicas, por ejemplo, a partir de la noción de descolonización en nuestros actos de conocer, piense por ejemplo en cómo pensarnos desde categorías latinoamericanas sin imponer las nociones o categorías europeas o sajonas en nuestros proyectos de investigación, un caso ejemplar es la noción de metodologías horizontales de Corona and Kaltmeier (2012), incluso, si planteamos los supuestos del pensamiento complejo no restringido acerca de la inter-disciplina o transdisciplinariedad, en la cual, podemos señalar el principio dialógico, que se refiere a la construcción de un espacio mental para tratar con situaciones contradictorias. Las consecuencias de los planteamientos anteriores salen del ámbito de la tradición aséptica del conocimiento y se colocan en la dimensión política, rompiendo el cercamiento relativo en las formas de conocer: conocimiento, política, ética y estética se encuentran. Sobre la complejidad restringida solo señalaremos que se trata de abordar la complejidad desde los supuestos de las lógicas pos-positivistas, es decir, reducirla y acortarla sobre aquella posición cartesiana de ideas claras y distintas, como lo expresa Morin (2008).

Las nociones señaladas más arriba -ontología, epistemología y metodología- son, para Bunge (1999), parte de las variables que configuran un campo de conocimiento. Expresadas en forma de preguntas tenemos, como señala De Gunther (2015): 1. ¿Quiénes investigan y cómo preparan a sus miembros? 2. ¿Cómo es la sociedad? 3. ¿Cuál es el universo del discurso? 4. ¿Cuál es el fundamento general o filosófico? 5. ¿Cuáles son sus teorías? 6. ¿Qué es un dato, hecho o fenómeno? 7. ¿Cuáles son sus objetivos? 8. ¿Cuáles son sus problemas? 9 ¿Cuáles son sus métodos? 10. ¿Cuál es la herencia del conocimiento acumulado? 11. ¿Cuáles son las disciplinas próximas? Tales preguntas permiten evaluar la consolidación o la exigua formación de un campo de conocimiento, tal tratamiento puede ser extensible a una disciplina. Asimismo, el reconocimiento de las variables señaladas, permiten generar una subrutina al interior del segundo supuesto, que permite avanzar hacia el tercer supuesto: el problema de investigación.

Sabemos que la investigación ayuda a responder preguntas -algunas urgentes, otras interesantes- colocadas en una disciplina o cómo la respuesta a tales preguntas requiere de dominios disciplinares diversos (interdisciplinarios e incluso transdisciplinarios en algunos casos ya articulados por otros/as). Preguntas que, sin duda, se colocan tanto en el desarrollo de las ciencias como en la limitación humana. Aspectos insoslayables en el acto de conocer y una invitación a la prudencia y mesura, sin que ello signifique constreñimiento alguno acerca de nuestros intereses de estudio o problemas de investigación.

Si en el principio está la pregunta, como ha señalado Gil Antón (2005), habría 
que reconocer que la pregunta se traduce como problema de investigación o interés de estudio y también como interés cognitivo, como señalamos más arriba. De ahí que el inicio de toda investigación comience "por el planteamiento de un problema...por una pregunta bien formulada que enraíza en una teoría o una tradición teórica y en el cuerpo del conocimiento acumulado pertinente a la cuestión..." (Cortés, Escobar, y González, 2008). Una afirmación que sugiere otras preguntas: ¿cómo se formula una pregunta o problema de investigación?, ¿cómo se sustenta adecuadamente? E incluso, otras inquietantes: ¿qué podemos hacer cuando la herencia del conocimiento disponible -la tradición teórica, metodológica y de interés cognitivo- es parcialmente útil o inútil para abordar nuestro problema de investigación? Lo anterior, sugiere un posicionamiento crítico acerca de esta tradición disciplinar para el desarrollo de la investigación, con ello nos referimos no sólo a los límites humanos para conciliar un proceso constructivo sujeto del error y la ilusión humana (Morin, 1999), sino también a la tradición de investigación lo ya sabido-, sobre el tema en cuestión en el interior de una disciplina.

La enunciación o planteamiento del problema se desdobla u operacionaliza, a su vez, tanto en el contexto como en los antecedentes de la investigación. En el primero de los casos se trata de dotar de sentido a las informaciones como lo ha enunciado Morin (1999), así como también, como lo hemos señalado en otro lugar (veáse De Gunther y Serrano, 2015), la contextualización genera cierta ignorancia sobre ciertas dimensiones de lo conocido, no por limitación de una capacidad humana, sino para dotar al sujeto de una optimización de la eficacia del funcionamiento cognitivo" (p. 31), con ello, ofrece tanto las condiciones para insertar el conocimiento actualizable como los límites de validez a través de una visión que religa y articula el fragmento y el todo de manera combinatoria (pensar globalmente, actuar localmente); mientras que los antecedentes dan cuenta de lo que se sabe, de lo ya sabido sobre el tema en cuestión, es decir, el llamado "estado de la cuestión", lo que deja como resultado el establecimiento de un vacío o ausencia -lo no sabido-, o una manera nueva de abordar un fenómeno sea en el ámbito metódico y otro.

Si bien esto se enuncia con cierta frescura, la subrutina enunciada en el supuesto dos, referido al conocimiento de las variables de un campo de conocimiento o una disciplina (como proceso de ida y vuelta), permite responder, no sin cierta exigencia, a la pregunta, ¿de dónde obtengo la información para la enunciación del problema? Se trata entonces de la identificación y selección de textos pertinentes al tema de interés, de la lectura cuidadosa de los mismos, de la identificación de los autores principales de la disciplina, del contexto de la investigación, de la experiencia personal sobre el mismo, del fondo formal o filosófico, de las teorías, de los fenómenos investigados, de los objetivos de las investigaciones, de los métodos empleados, de la creación de una base de datos contrastable y comparable entre las investigaciones y textos revisados y la experiencia propia. En todo caso, convendría poner atención en lo señalado por Swedberg (2014), acerca de que se ha alcanzado un alto dominio en cuestiones metodológicas referidas a métodos y técnicas, pero poco con relación al proceso de teorización como proceso y no sólo como producto: teoría/teorización; una cuestión que podemos extender a los dominios del problema de investigación: de la problematización al problema; que bien podemos denominar contexto de descubrimiento y contexto de justificación (Popper, Adorno, Dahrendorf, y Habermas, 2008) e incluso, ante nuestra 
perplejidad, recuperar la noción de imaginación sociológica a la que alude Mills (2004):

la imaginación sociológica consiste, en una parte considerable, en la capacidad de pasar de una perspectiva a otra y en el proceso de formar una opinión adecuada de una sociedad total y de sus componentes. Es esa imaginación, naturalmente, lo que separa al investigador social del mero técnico (p. 222).

De la misma manera, una vez clarificado el problema o pregunta de investigación, nos encontramos ante el cuarto supuesto: el orientador teórico. Se trata del lente a través del cual observar o interpretar los "datos" o informaciones obtenidos, recordemos que las informaciones cobran sentido a partir del sistema teórico desde el cual se interpretan, como lo ha señalado González Rey (2007) y, como es posible inferir, es susceptible de encontrarse en la subrutina enunciada en el supuesto dos, las variables del campo de conocimiento, en nuestra base de datos, en las fichas de teorías, que han sido empleadas para tratar problemas semejantes, e incluso, proponer la inadecuación de las mismas y salir a la búsqueda de otros posicionamientos o de su construcción. Estas orientaciones permiten dar cuenta de nuestra contribución a la teoría.

La búsqueda de respuesta a la pregunta de investigación, nos dirige ahora al quinto supuesto, la enunciación de la metodología, la cual requiere de un método de investigación adecuado, en este sentido la observación de Tulmin acerca de la adecuación del método al problema resulte un índice útil a tomar en cuenta (como se cita en Marradi, Archenti, y Piovani, 2007), ya que cada problema de investigación construye su propio método. Incluso, como señala Gutiérrez (2013), en su posicionamiento epistémico-político: No hay un método, hay métodos, tantos como objetos de estudio sea posible pen- sar, construir. El sujeto debe romper con la pretendida preexistencia del "método", igual que se debe romper con la teoría como un marco conceptual que todo lo explica y, de la misma forma, romper con las aproximaciones meramente empiristas e instrumentales (p. 10).

Si asumimos la existencia de métodos y no del "Método" -con mayúscula-, podríamos señalar como lo hace Baofu (2008), que las disciplinas poseen diferentes metodologías para el estudio de la realidad y cada metodología permitiría, a la vez, más de un método o técnica de investigación, bajo los cuales subyacen constructos ontológicos, epistemológicos y metodológicos. Tales aspectos se pueden localizar, otra vez, en la subrutina enunciada en el supuesto dos, las variables del campo de conocimiento. Además de sus particularidades sea la descripción del método, los objetivos, las preguntas, las hipótesis, las dimensiones, las variables y los indicadores.

En este momento podemos enunciar que el aprendizaje de la metodología no sólo trata del entrenamiento instrumental ni únicamente de la compresión ontológica, epistemológica que la sostiene, sino de un tipo de conocimiento que contiene ambos supuestos, y la experiencia personal, en cuyo caso, permiten actualizar como orientador de nuestra reflexión el planteamiento de Bruschi:

una metodología sin reflexión sobre los fines, condiciones y límites del conocimiento científico es sólo una tecnología o práctica fuera del control de quien la ejerce, mientras que, si abandona el lado tecnológico o práctico, se convierte en reflexión filosófica, sin injerencia en las actividades de investigación. De ahí que, a toda actividad metódica (técnico-práctica) subyazcan soluciones metodológicas y, a estas, respuestas a cuestiones epistemológicas (como se cita en Marradi et al., 
2007) y, agregamos a estas, ontológicas, las cuales tienen consecuencias en nuestras formas de conocer, más adelante volveremos con esto.

Lo anterior permite desprender el rol prescriptivo o normativo de la metodología y, al menos, una implicación liberadora: si toda metodología elabora o sigue procedimientos específicos (métodos, técnicas) aceptables, empleados en una ciencia particular o por un grupo de investigadores específico, debemos recordar que tales elaboraciones no son modelos abstractos, sino que siguen invariablemente orientaciones conscientes o inconscientes de quien las elabora. Es decir, hay una toma de decisiones, una posición crítica, una toma de decisiones asociadas a ellas, sean o no racionales, que implican que en la producción científica no sólo está en juego la ciencia, sino, además, el prestigio del investigador, la lucha por la apropiación del capital simbólico o cultural para su continuidad o transformación, las luchas entre los miembros del campo, entre otras cuestiones que ha apuntado Bourdieu (2002).

De ahí que las críticas tanto internas como externas al paradigma dominante a las que aluden Guba y Lincoln (1994) y Cortes (2008), no deben ser sobrestimadas y nos pronunciemos por su clarificación y adecuación contextual y, con ello, la incorporación de la experiencia propia y ajena en nuestro proceder: "Lo esencial del concepto de método está... en la elección de las técnicas a aplicar, en la capacidad de modificar técnicas existentes, adaptándolas a los problemas propios, y de imaginar técnicas nuevas" (Marradi et al., 2007, p. 56), lo cual contribuye no sólo la reflexión metodológica; sino nuestra comprensión de lo que sea un paradigma en su sentido amplio.

Las ideas anteriores conducen a plantear que la formación científica requiere no sólo la adquisición de un dominio técnico específico; sino además de otras dimensiones sean cognoscitivas, críticas, reflexivas y creativas, que permitan reconocer el alcance de las cuestiones socio-culturales y políticas en el entramado de la investigación.

Recordemos que, como señala Ratcliffe y González del Valle (2000), que si bien los procesos de investigación tienden a centrarse en el método como indicador de rigor, con ello olvidan que el rigor de la investigación se encuentra ligado a todo el proceso de su desarrollo: es sistémico. De ahí que la investigación requiera pensarse en un marco amplio, que dé cuenta de sus bases, sus procesos, sus cuestiones éticas y su producción textual. Un ejercicio de construcción crítica permanente. Dicho de otra manera, la investigación se nos presenta como una exigencia creativa y crítica del investigador, como exploración, diálogo y construcción cognitiva y experiencial con respecto a su tema y al campo de conocimiento al que pertenece, así como viaje de exploración y diálogo vivencial entre y a través de los campos de conocimiento. Se trata de pensar lo menos reductoramente posible la investigación (Morin, 1983).

Conviene, antes de cerrar, enunciar al menos dos supuestos más que subyacen a lo que enseñamos y al cómo lo hacemos, el sexto que orienta la presentación de resultados, los cuales se organizan en función de los objetivos establecidos, que requieren formas adecuadas de presentación, por lo que conviene revisar nuestra subrutina para conocer cómo otros presentan sus resultados y, el séptimo, comprender la tesis como un género dentro de la esfera de la actividad de investigación, el cual como señala Llamas Saíz (2014), "produce situaciones regulares que dan lugar a textos de características lingüísticas y estructurales similares, sea un artículo científico, una tesis doctoral, un resumen, un trabajo de fin de grado, una ponencia" (p. 15), es pues una acción social, está condicionada 
por el contexto donde se ejerce, en este caso la universidad y las disciplinas desde donde se escribe. Sin embargo, si bien la escritura académica está condicionada en su puesta en práctica, también transforma sus condicionantes a través de la práctica (Llamas Saíz, 2014), de ahí que podamos pensarla como obra abierta o, para decirlo con Lotman, la escritura construye mundos existentes o posibles (como se cita en como se cita en Bengochea y Atorresi, 2010) y, agregamos, la escritura de un texto no solo trata de una habilidad cognitiva-instrumental, sino que, lo que hace al texto, su complejidad sea el género, el estilo, el formato, el soporte material, la función, el modo de circulación etcétera, exige una comprensión amplia para su producción.

Finalmente, señalamos que no hemos tocado aquí las cuestiones relativas al espacio-tiempo a las que alude Valencia (2007), ni tampoco el detallado trabajo de construcción de objetos de conocimiento enunciado por Gutierrez (2013), aspectos que si bien los tocamos de soslayo requieren de un tratamiento en extenso de los mismos.

\section{Consideraciones finales}

En los apartados anteriores hemos mostrado primero la investigación como una esfera de la actividad humana generada por la universidad y diferenciada de otras actividades al interior de la universidad, es pues un género. También colocamos, la reflexividad metodológica como una herramienta útil para mostrar los supuestos $u$ orientadores que subyacen a la enseñanza de la investigación en el posgrado en Humanidades, estudios en artes de la Universidad de Sonora y, ofrecer a la vez, una respuesta a la pregunta central qué enseñamos y cómo enseñamos a investigar en el entramado de los distintos modos de saber disponibles sea en las ciencias naturales, las ciencias sociales, las humanidades y las artes, así como también, en las ciencias de la complejidad sea restringida o no restringida y en los estudios culturales (Morin, 2008; Wallerstein, 2002), tal pregunta la colocamos en la dimensión del profesorado como uno de los actores centrales de este proceso en quienes enseñamos y dirigimos trabajos de investigación.

Tal ejercicio nos permitió establecer un conjunto de supuestos que permiten comprender los elementos que subyacen a nuestra enseñanza de la investigación, los cuales han sido enunciados progresivamente y que ahora esquematizamos. Primer supuesto, la orientación curricular, constituye el punto de partida para la enseñanza de la investigación. Segundo supuesto: el reconocimiento y conocimiento del campo de conocimiento y de las disciplinas afines. Subrutina, derivada del conocimiento de las variables que constituyen el campo de conocimiento y de las disciplinas afines. Tercer supuesto, el problema de investigación. Cuarto supuesto, el orientador teórico. Quinto supuesto, la enunciación de la metodología. Sexto supuesto la presentación de resultados. Séptimo supuesto, comprender la tesis como un género de escritura.

Los supuestos enunciados en el párrafo anterior, avivan la hipótesis de trabajo que orientan este trabajo, la cual ha sido enunciada por Marradi, Archenti, y Piovani, (2007): una metodología sin reflexión sobre los fines, condiciones y límites del conocimiento es sólo una tecnología o práctica fuera del control de quien la ejerce, mientras que, si abandona el lado tecnológico o práctico, se convierte en reflexión filosófica, sin injerencia en las actividades de investigación. De ahí que, a toda actividad metódica (técnico-práctica) subyazcan soluciones metodológicas $\mathrm{y}$, a estas, respuestas a cuestiones epistemológicas y, agregamos, a estas, ontológicas, las cuales tienen consecuencias en nuestras formas de conocer. De ahí que el epígrafe que acompaña el texto cobre sentido al final de este trabajo. 


\section{Bibliografía}

Bajtin, M. M. (1999). Estética de la creación verbal (T. Bubnova, Trad. 10ma ed.). México: Fondo de Cultura Económica.

Baofu, P. (2008). The Future of Post-Human Knowledge. A Preface to a new Theory of Methodology and Ontology. Oxford · England: Chandos Publishing.

Bengochea, R., y Atorresi, A. (2010). Escritura: un estudio de las habilidades de los estudiantes de América Latina y el Caribe. Santiago de Chile, CL: B - UNESCO.

Bourdieu, P. (2002). Las reglas del arte: Génesis y estructura del campo literario (T. Kauf, Trad. 3ra ed.). Barcelona: Anagrama.

Bunge, M. (1999). Buscar la filosofía en las ciencias sociales (T. Aguilar., Trad.). México: Fondo de Cultura Económica.

Castelló Badía, M. (2014). Los retos actuales de la alfabetización académica: estado de la cuestión y últimas investigaciones. En I. Ballano y I. Muñoz (Eds.), Escribir en el contexto académico (pp. 153-176). Bilbao, ES: Publicaciones de la Universidad de Deusto.

Corona, S., y Kaltmeier. (2012). El diálogo. Metodología horizontales en Ciencias Sociales y Culturales (1a ed. Vol. 17). México: Gedisa.

Cortés, F. (2008). Algunos aspectos de la controversia entre la investigación cualitativa y la investigación cuantitativa. En F. Cortés, A. Escobar, y M. González (Eds.), Método científico y política social. A propósito de las evaluaciones cualitativas de programas sociales (1a ed., pp. 27-58). México: Colegio de México.

Cortés, F., Escobar, A., y González, M. (2008). Método científico y política social. A propósito de las evaluaciones cualitativas de programas sociales. México: Colegio de México.

De Gunther, L. (2015). Aportes para el abordaje del campo de conocimiento de las artes de la Universidad de Sonora: las dimensiones formal, social y política de su constitución. En C. Hurtado, R. Lozano, L. De Gunther, y U. Mazariegos (Eds.), Una Visión Interdisciplinaria del Arte (1a ed., pp. 44-62). México: Universidad de Sonora.

De Gunther, L., y Serrano, F. (2015). La Hermenéutica analógica-icónica: una herramienta para la investigación en las artes visuales. Arte entre paréntesis (1), 28-35.

Gabriel, M. (2015). why the world does not exist (G. Moss, Trad.). USA: Polity Press.

García, R. (2006). Sistemas complejos: Concepto, método y fundamentación epistemológica de la investigación interdisciplinaria. (1era. ed.). Barcelona: Gedisa.

Gil Anton, M. (2004). ¿Ciencias duras y ciencias blandas? Una falsa dicotomía. Contaduría y Adminstración, 213(3), 151-164.

Gil Antón, M. (2005). Max Weber: el valor de las preguntas. Sociológica, 20(59), 93-114.

González Rey, F. L. (2007). Investigación cualitativa y subjetividad : los procesos de construcción de la información. México: McGraw-Hill Interamericana.

Guba, E. G., y Lincoln, Y. S. (1994). Competing Paradigms in Qualitative Research. En N. Denzin y Y. Lincoln (Eds.), Handbook of Qualitative Research (pp. 105-117). Newbury Park: Sage.

Gutierrez, D. (2013). Consideraciones epistemológicas para la construcción de objetos de investigación en Ciencias Sociales y Humanidades. México: Universidad de Sonora.

Leal, F. (2003). ¿Qué es crítico? Apuntes para la historia de un término. Revista Mexicana de Investigación Educativa, 8(17), 245-261. 
Llamas Saíz, C. (2014). Escribir en el contexto académico: conocimientos y estrategias. En I. Ballano y I. Muñoz (Eds.), Escribir en el contexto académico (pp. 13-33). Bilbao, ES: Publicaciones de la Universidad de Deusto.

López, S., Alvarado, A., y Mungaray, A. (2011). La comunicación de la ciencia a través de articulos científicos (1a ed.). México: Universidad de Occidente-Juan Pablos Editor.

Mari Mut, J. A. (2013). Manual de Redacción Científica. Retrieved from https:// ia601305.us.archive.org/22/items/ManualDeRedaccionCientifica/Manual\%20 de\%20Redacci\%C3\%B3n\%20Cient\%C3\%ADfica.pdf

Marradi, A., Archenti, N., y Piovani, J. (2007). Metodología de las ciencias sociales (1era ed.). Buenos Aires: Emecé.

Mills, C. W., y Torner, F. M. (2004). La imaginación sociológica. México D.F. (México): Fondo de Cultura Económica.

Morin, E. (1983). El método II: La vida de la vida (A. Sánchez, Trad. 5ta. ed. Vol. 2). Madrid: Cátedra.

Morin, E. (1999). Los 7 saberes necesarios para la educación del futuro (M. Vallejo-Gómez y F. Girard, Trad.). París: Unesco.

Morin, E. (2008). Complejidad restringida y complejidad generalizada. Universidad Autónoma de Nuevo León: Catedra Itinerante Edgar Morin (Unesco)-IIPC-USALUANL.

Pérez, D., y Rodríguez , L. (Eds.). (2011). Explicar y comprender (1a ed.). México: Plaza y Valdes.

Piovani, J. (2016). Reflexiones sobre el proceso de investigación social. En C. Gallegos, A. Mejía, y Y. Paredes (Eds.), ¿Cómo investigamos? ¿Cómo enseñamos a investigar? Tomo IV. En memoriam Hugo Zemelman Merino (pp. 47-64). México: Unam.

Popper, Adorno, Dahrendorf, y Habermas. (2008). Lógica de las ciencias sociales (J. Muñoz, Trad.). México: Colofón.

Ratcliffe, J. W., y González del Valle, A. (2000). El rigor en la investigación de la salud. En C. A. Denman y J. A. Haro (Eds.), Por los rincones: Antología de métodos de investigación cualitativos en la investigación social (pp. 57-111). Hermosillo: Colegio de Sonora.

Swedberg, R. (Ed.) (2014). Theorizing in social science : the context of discovery. United States of America: Stanford University Press.

Valencia, G. (2007). Entre Cronos y Kayros. Las formas del tiempo sociohistórico. España: Anthropos.

Valles, M. S. (2009). Técnicas cualitativas de investigación social : reflexión metodológica y práctica profesional. Madrid: Síntesis.

Vargas, W. L. (2009). Acerca de la escritura de artículos científicos. Ciencia e Ingeniería Neogranadina, 14(50-61), 1-15. Retrieved from http://site.ebrary.com/lib/ usonsp/docDetail.action?doclD=10345017.

Wallerstein, I. (2007). Impensar las ciencias sociales. Límites de los paradigmas decimonónicos (S. Guardado, Trad. 5a ed.). México: Siglo XXI editores.

Wallerstein, I., Juma. Calestou, Fox Keller, E., Jürgen, K., Lecourt, D., Mudimbe, V., . . . Lee, R. (1996). Abrir las ciencias sociales. Informe de la Comisión Gulbenkian para la reestructuración de las ciencias sociales (S. Mastrángelo, Trad. 10ma ed.). México: Siglo XXI editores. 